\title{
On Marginal Effects in Semiparametric Censored Regression Models
}

\author{
Bo E. Honoré*
}

September 13, 2008

\section{Introduction}

It is often argued that estimation of semiparametric censored regression models such as the ones in Powell (1984), Powell (1986), Honoré (1992) and Chen and Khan (2000) do not deliver marginal effects, even when the model is correctly specified (see for example the concluding paragraph of Greene (1999)). The following shows that this is incorrect.

Consider the censored regression model,

$$
y=\max \left\{0, x^{\prime} \beta+\varepsilon\right\}
$$

where $x$ is a set of explanatory variables and $\varepsilon$ is an error-term. Greene (1999) showed that ${ }^{1}$

$$
\frac{\partial E[y \mid x]}{\partial x_{j}}=\beta_{j} P(y>0 \mid x)=\beta_{j} P(\{y \text { not censored }\} \mid x)
$$

when $x$ and $\varepsilon$ are independent, and where $x_{j}$ denotes the $j$ 'th element of $x$. It follows from (2) that the average derivative is

$$
E\left[\frac{\partial E[y \mid x]}{\partial x_{j}}\right]=\beta_{j} P(y>0)=\beta_{j} P(\{y \text { not censored }\})
$$

When $x$ and $\varepsilon$ are not independent (2) and (3) will generally not be correct, and calculation of $\frac{\partial E[y \mid x]}{\partial x_{j}}$ or $E\left[\frac{\partial E[y \mid x]}{\partial x_{j}}\right]$ will require additional knowledge about the distribution of $\varepsilon$ given $x$. The

*Mailing Address: Department of Economics, Princeton University, Princeton, NJ 08544-1021. Email: honore@Princeton.edu. This research was supported by the NSF Grant No. SES-0417895 to Princeton University, The Gregory C. Chow Econometric Research Program at Princeton University.

${ }^{1}$ The calculations in the note implicitly assume that one is interested in the censored dependent variable. This is typically the case when the censoring is caused by "corner solutions" (see Wooldridge (2001)). When the censoring is due to "data censoring" (such as in Chay and Honore (1998) and Hu (2002)), one would be interested in the marginal effect of $x$ on the uncensored dependent variable, $x^{\prime} \beta+\varepsilon$. This is just $\beta$. 
point of this note is to show that $\beta_{j} P(\{y$ not censored $\})$ is still an interesting marginal effects in that case.

When $x$ and $\varepsilon$ are not independent, the marginal effect

$$
E\left[\frac{\partial E[y \mid x]}{\partial x_{j}}\right]
$$

implicitly assumes that (the distribution of) $\varepsilon$ in (1) changes if a policy maker changes $x$. This is not always the interesting thought-experiment. Suppose, for example, that $\varepsilon$ and $x$ are dependent because the former includes a "fixed" effect. In that case, one would want to know the effect of exogenously changing $x$, holding the fixed effect constant. The same if true one thinks of $\varepsilon$ as an explanatory variable that happens to be unobserved. In that case, we would want to hold $\varepsilon$ as well as the other explanatory constant fixed when evaluating the marginal effect of $x_{j}$. This is similar to the argument for why one would be interested in instrumental variables estimation and not just the coefficients in the projection of a dependent variable on a set of explanatory variables. See, for example, Stock and Watson (2007). ${ }^{2}$ We therefore define the marginal effect

$$
\lim _{\delta \rightarrow 0} E\left[\frac{\max \left\{0, x^{\prime} \beta+\delta \beta_{j}+\varepsilon\right\}-\max \left\{0, x^{\prime} \beta+\varepsilon\right\}}{\delta}\right] .
$$

We interpret this as the average marginal effect of changing the $j$ 'th element of $x$, holding all other variables constant.

Below we show as a special case that

$$
\lim _{\delta \rightarrow 0} E\left[\frac{\max \left\{0, x^{\prime} \beta+\delta \beta_{j}+\varepsilon\right\}-\max \left\{0, x^{\prime} \beta+\varepsilon\right\}}{\delta}\right]=\beta_{j} P(y>0)
$$

In other words, $\beta_{j} P(\{y$ not censored $\})$ is an interesting marginal effect even if $x$ and $\varepsilon$ are not independent.

\section{The result}

The general result holds for models with two-sided as well as one-sided censoring and it does not matter whether the censoring variables are random of fixed (provided that the thought-experiment does not change the censoring points).

To simplify the notation, define

$$
\operatorname{mami}\left\{a_{1}, x, a_{2}\right\}=\left\{\begin{array}{ccc}
a_{1} & \text { if } & x<a_{1} \\
x & \text { if } & a_{1} \leq x \leq a_{2} \\
a_{2} & \text { if } & x>a_{2}
\end{array}\right.
$$

\footnotetext{
${ }^{2}$ It is also consistent with the interpretation of the regression coefficients in a linear panel data model with fixed effects, as the marginal effects of the regressors holding the fixed effect constant.
} 
The model that we will consider is

$$
y=\left\{\begin{array}{c}
L \\
x^{\prime} \beta+\varepsilon \\
U
\end{array} \quad \equiv \operatorname{mami}\left\{L, x^{\prime} \beta+\varepsilon, U\right\}\right.
$$

where $L \leq U$ and $(L, U, \varepsilon)$ is allowed to be random and depend on $x$. $L$ is allowed to be $-\infty$ and $U$ is allowed to be $\infty$. This has the censored regression model with fixed one-sided censoring at 0 (equation (1)) as a special case, and in a panel data setting it does not matter whether one thinks of $\varepsilon$ as including a "fixed" effect.

In the model (6), we will be interested in

$$
\lim _{\delta \rightarrow 0} E\left[\frac{\operatorname{mami}\left\{L, x^{\prime} \beta+\delta \beta_{j}+\varepsilon, U\right\}-\operatorname{mami}\left\{L, x^{\prime} \beta+\varepsilon, U\right\}}{\delta}\right]
$$

where the distribution is taken over the distribution of $(L, U, \varepsilon)$.

We first note that for $v>0$,

$$
\begin{gathered}
\operatorname{mami}\{L, z+\nu, U\}-\operatorname{mami}\{L, z, U\}=0 \quad \text { for } \quad z \leq L-v \\
0 \leq \operatorname{mami}\{L, z+\nu, U\}-\operatorname{mami}\{L, z, U\} \leq v \quad \text { for } \quad L-v \leq z \leq L \\
\operatorname{mami}\{L, z+\nu, U\}-\operatorname{mami}\{L, z, U\}=v \text { for } \quad L \leq z \leq U-v \\
0 \leq \operatorname{mami}\{L, z+\nu, U\}-\operatorname{mami}\{L, z, U\} \leq v \text { for } \quad U-v \leq z \leq U \\
\operatorname{mami}\{L, z+\nu, U\}-\operatorname{mami}\{L, z, U\}=0 \text { for } \quad U \leq z
\end{gathered}
$$

So

$$
v \cdot 1\{L \leq z \leq U-v\} \leq \operatorname{mami}\{L, z+\nu, U\}-\operatorname{mami}\{L, z, U\} \leq v \cdot 1\{L-v \leq z \leq U\}
$$

and

$$
\begin{aligned}
& -v \cdot(1\{U-v \leq z \leq U+v\}+1\{L-v \leq z \leq L+v\}) \\
\leq & (\operatorname{mami}\{L, z+\nu, U\}-\operatorname{mami}\{L, z, U\})-v \cdot 1\{L \leq z \leq U\} \\
\leq & v \cdot(1\{U-v \leq z \leq U+v\}+1\{L-v \leq z \leq L+v\})
\end{aligned}
$$

where the inequalities are deliberately made less tight in order to simplify the expressions below.

Likewise when $v<0$

$$
\begin{aligned}
& v \cdot(1\{U+v \leq z \leq U-v\}+1\{L+v \leq z \leq L-v\}) \\
\leq & (\operatorname{mami}\{L, z+\nu, U\}-\operatorname{mami}\{L, z, U\})-v \cdot 1\{L \leq z \leq U\} \\
\leq & -v \cdot(1\{U+v \leq z \leq U-v\}+1\{L+v \leq z \leq L-v\})
\end{aligned}
$$


Either way

$$
\begin{aligned}
& -|v| \cdot(1\{|U-z| \leq|v|\}+1\{|L-z| \leq|v|\}) \\
\leq & (\operatorname{mami}\{L, z+\nu, U\}-\operatorname{mami}\{L, z, U\})-v \cdot 1\{L \leq z \leq U\} \\
\leq & |v| \cdot(1\{|U-z| \leq|v|\}+1\{|L-z| \leq|v|\})
\end{aligned}
$$

This implies that

$$
\begin{aligned}
& -\left|\beta_{j}\right| \cdot\left(1\left\{\left|U-x^{\prime} \beta-\varepsilon\right| \leq|\delta| \cdot\left|\beta_{j}\right|\right\}+1\left\{\left|L-x^{\prime} \beta-\varepsilon\right| \leq|\delta| \cdot\left|\beta_{j}\right|\right\}\right) \\
\leq & \frac{\operatorname{mami}\left\{L, x^{\prime} \beta+\delta \beta_{j}+\varepsilon, U\right\}-\operatorname{mami}\left\{L, x^{\prime} \beta+\varepsilon, U\right\}}{\delta}-\beta_{j} 1\left\{L \leq x^{\prime} \beta+\varepsilon \leq U\right\} \\
\leq & \left|\beta_{j}\right| \cdot\left(1\left\{\left|U-x^{\prime} \beta-\varepsilon\right| \leq|\delta| \cdot\left|\beta_{j}\right|\right\}+1\left\{\left|L-x^{\prime} \beta-\varepsilon\right| \leq|\delta| \cdot\left|\beta_{j}\right|\right\}\right)
\end{aligned}
$$

so

$$
\begin{aligned}
& -\left|\beta_{j}\right| \cdot\left(P\left(\left|U-x^{\prime} \beta-\varepsilon\right| \leq|\delta| \cdot\left|\beta_{j}\right|\right)+P\left(\left|L-x^{\prime} \beta-\varepsilon\right| \leq|\delta| \cdot\left|\beta_{j}\right|\right)\right) \\
\leq & E\left[\frac{\operatorname{mami}\left\{L, x^{\prime} \beta+\delta \beta_{j}+\varepsilon, U\right\}-\operatorname{mami}\left\{L, x^{\prime} \beta+\varepsilon, U\right\}}{\delta}\right]-\beta_{j} P\left(L \leq x^{\prime} \beta+\varepsilon \leq U\right) \\
\leq & \left|\beta_{j}\right| \cdot\left(P\left(\left|U-x^{\prime} \beta-\varepsilon\right| \leq|\delta| \cdot\left|\beta_{j}\right|\right)+P\left(\left|L-x^{\prime} \beta-\varepsilon\right| \leq|\delta| \cdot\left|\beta_{j}\right|\right)\right)
\end{aligned}
$$

so if $U-x^{\prime} \beta-\varepsilon$ and $L-x^{\prime} \beta-\varepsilon$ both have densities in a neighborhood of 0 , then

$$
\begin{aligned}
& \lim _{\delta \rightarrow 0} E\left[\frac{\operatorname{mami}\left\{L, x^{\prime} \beta+\delta \beta_{j}+\varepsilon, U\right\}-\operatorname{mami}\left\{L, x^{\prime} \beta+\varepsilon, U\right\}}{\delta}\right] \\
= & \beta_{j} P\left(L \leq x^{\prime} \beta+\varepsilon \leq U\right) \\
= & \beta_{j} P(\{y \text { not censored }\})
\end{aligned}
$$

In other words, the quantity $\beta_{j} P(\{y$ not censored $\})$ gives the average marginal effect on $y$ of exogenously changing $x_{j}$, holding $(L, U, \varepsilon)$ fixed.

We note that the same argument can be used to get the average marginal effect for some subset of the population. Specifically,

$\lim _{\delta \rightarrow 0} E\left[\frac{\operatorname{mami}\left\{L, x^{\prime} \beta+\delta \beta_{j}+\varepsilon, U\right\}-\operatorname{mami}\left\{L, x^{\prime} \beta+\varepsilon, U\right\}}{\delta} \mid x \in \mathcal{A}\right]=\beta_{j} P(\{y$ not censored $\} \mid x \in \mathcal{A})$

\section{Estimation}

Estimators of $\beta$ in a censored regression model have been proposed under a number of assumptions. These are almost always asymptotically normally distributed and it is typically straightforward to used this to derive the asymptotic distribution of the marginal effect discussed in the previous 
section. Consider for example the censored absolute deviations estimator proposed by Powell (1984). This estimator applies to (1) under the assumption that median $(\varepsilon \mid x)=0$. The estimator is defined by

$$
\widehat{\beta}=\arg \min _{b} \sum_{i=1}^{n}\left|y_{i}-\max \left\{0, x_{i}^{\prime} b\right\}\right|,
$$

where $\left(y_{i}, x_{i}^{\prime}\right)$ denoted the values of $y$ and $x$ for the $i$ 'th observation. The asymptotic distribution is then found from the approximate moment conditions

$$
\frac{1}{n} \sum_{i=1}^{n} 1\left\{x_{i}^{\prime} \widehat{\beta}>0\right\} \operatorname{sign}\left(y_{i}-x_{i}^{\prime} \widehat{\beta}\right) x_{i} \approx 0
$$

Generically, estimators of $\beta$ typically satisfy conditions of the type

$$
\frac{1}{n} \sum_{i=1}^{n} m\left(z_{i}, \widehat{\beta}\right) \approx 0
$$

where $z_{i}$ denotes all the data for observation $i .^{3}$

Letting $\kappa$ denote the vector of marginal effects in (5) and augmenting (9) by

$$
\frac{1}{n} \sum_{i=1}^{n}\left(1\left\{y_{i}>0\right\} \widehat{\beta}-\widehat{\kappa}\right)=0,
$$

we get (under random sampling and subject to weak regularity conditions)

$$
\sqrt{n}\left(\left(\begin{array}{c}
\widehat{\beta} \\
\widehat{\kappa}
\end{array}\right)-\left(\begin{array}{c}
\beta \\
\kappa
\end{array}\right)\right) \simeq N\left(0, \Gamma^{-1} V \Gamma^{-1}\right)
$$

where

$$
\Gamma=\left(\begin{array}{cc}
\frac{\partial E\left[m\left(z_{i}, \beta\right)\right]}{\partial \beta^{\prime}} & 0 \\
E\left[1\left\{y_{i} \text { uncensored }\right\}\right. & I]
\end{array}\right)
$$

and

$$
V=
$$

$\left(\begin{array}{cc}E\left[m\left(z_{i}, \beta\right) m\left(z_{i}, \beta\right)^{\prime}\right] & E\left[m\left(z_{i}, \beta\right)\left(1\left\{y_{i} \text { uncensored }\right\} \beta-\kappa\right)^{\prime}\right] \\ E\left[\left(1\left\{y_{i} \text { uncensored }\right\} \beta-\kappa\right) m\left(z_{i}, \beta\right)^{\prime}\right] & E\left[\left(1\left\{y_{i} \text { uncensored }\right\} \beta-\kappa\right)\left(1\left\{y_{i} \text { uncensored }\right\} \beta^{\prime}-\kappa^{\prime}\right)\right]\end{array}\right)$

For Powell's CLAD estimator,

$$
\Gamma=\left(\begin{array}{cc}
2 E\left[f_{\varepsilon \mid x}\left(0 \mid x_{i}\right) x_{i} x_{i}^{\prime}\right] & 0 \\
E\left[1\left\{y_{i}>0\right\} I\right] & I
\end{array}\right)
$$

so

$$
\Gamma^{-1}=\left(\begin{array}{cc}
\left(2 E\left[f_{\varepsilon \mid x}\left(0 \mid x_{i}\right) x_{i} x_{i}^{\prime}\right]\right)^{-1} & 0 \\
-E\left[1\left\{y_{i}>0\right\} I\right]\left(2 E\left[f_{\varepsilon \mid x}\left(0 \mid x_{i}\right) x_{i} x_{i}^{\prime}\right]\right)^{-1} & I
\end{array}\right)
$$

and

$$
V=\left(\begin{array}{cc}
E\left[1\left\{x_{i}^{\prime} \beta>0\right\} x_{i} x_{i}^{\prime}\right] & E\left[1\left\{x_{i}^{\prime} \beta>0\right\}\left(1\left\{y_{i}>0\right\} x_{i} \beta^{\prime}-x_{i} \kappa^{\prime}\right)\right] \\
E\left[1\left\{x_{i}^{\prime} \beta>0\right\}\left(1\left\{y_{i}>0\right\} x_{i} \beta^{\prime}-x_{i} \kappa^{\prime}\right)\right]^{\prime} & E\left[\left(1\left\{y_{i}>0\right\} \beta-\kappa\right)\left(1\left\{y_{i}>0\right\} \beta^{\prime}-\kappa^{\prime}\right)\right]
\end{array}\right)
$$

\footnotetext{
${ }^{3}$ Note that this notation allows for cross sectional as well as panel data.
} 


\section{Conclusion}

This note illustrates that the typical parameter, $\beta$, in a censored regression model can be used to calculate an interesting marginal effect even when the errors in the model and the explanatory variables are not independent. The result is relevant for cross sectional models such at the ones considered in Powell (1984), Powell (1986) and Chen and Khan (2000), as well as for panel data models such as the ones in Honoré (1992) and Alan and Leth-Petersen (2006), and it applies with fixed as well as with random censoring.

\section{References}

Alan, S., and S. Leth-Petersen (2006): "Tax Incentives and Household Portfolios: A Panel Data Analysis," Center for Applied Microeconometrics, University of Copenhagen, Working paper number 2006-13.

Chay, K. Y., and B. E. Honore (1998): "Estimation of Semiparametric Censored Regression Models: An Application to Changes in Black-White Earnings Inequality during the 1960s," Journal of Human Resources, 33(1), 4-38.

Chen, S., And S. Khan (2000): "Estimating censored regression models in the presence of nonparametric multiplicative heteroskedasticity," Journal of Econometrics, 98, 283-316.

Greene, W. (1999): "Marginal effects in the censored regression model," Economics Letters, 64(1), 43-49.

Honoré, B. E. (1992): "Trimmed LAD and Least Squares Estimation of Truncated and Censored Regression Models with Fixed Effects," Econometrica, 60, 533-565.

Hu, L. (2002): "Estimation of a Censored Dynamic Panel Data Model," Econometrica, 70(6), 2499-2517.

Powell, J. L. (1984): "Least Absolute Deviations Estimation for the Censored Regression Model," Journal of Econometrics, 25(3), 303-25.

- (1986): "Symmetrically Trimmed Least Squares Estimation for Tobit Models," Econometrica, 54, 1435-1460.

Stock, J., And M. Watson (2007): Introduction to Econometrics. Addison-Wesley, 2 edn. 
Wooldridge, J. M. (2001): Econometric Analysis of Cross Section and Panel Data. The MIT Press. 